\title{
Pelvic Enchondroma: A Rare Location And Cause Of Dyspareunia And Infertility.
}

\author{
Onuoha K.M, Idowu B.S, Orimolade E.A, Makinde O.N \\ Departments of Orthopaedics and Trauma, Obstetrics and Gynaecology, \\ Obafemi Awolowo University Teaching Hospitals Complex, Ile-Ife, Osun state, Nigeria.
}

\begin{abstract}
Enchondromas are rarely occurring tumours in the pelvis. They have predilection for the phalanges and their occurrence in male and female are equal. We present a case of a 32 year old nulliparous who had history of painless pelvic mass and deep dyspareunia that precluded her from having satisfying coitus hence avoidance of her husband and subsequent infertility. The tumour was resected via Maylard abdominal incision and access was gained into retro-pubic space of Retzius avoiding the bladder and its neck structures. Haemostasis was secured and reconstruction plate and screws were used in stabilising the superior pubic ramus. She recuperated well and currently on follow up in Orthopaedic out-patient department.
\end{abstract}

Keywords: Pelvic Enchondroma, Dyspareunia, Infertility.

\section{Introduction}

Chondromas are benign lesions of hyaline cartilage. They are common, and affect all age groups. Chondromas usually, are asymptomatic and frequently are discovered incidentally during an unrelated radiographic examination. This group of tumours usually arise in the medullary canal, where they are referred to as Enchondromas, rarely; they may arise on the surface of the bone, where they are referred to as periosteal chondromas or juxtacortical chondromas. The judicious use of computed tomography, magnetic resonance imaging, and nuclear medicine in conjunction with appropriate clinical data allows the clinician to establish the correct diagnosis of benign or malignant medullary chondroid lesion in the majority of cases.(1)

Peak incidence of Enchondromas occurs in the $3^{\text {rd }}$ decade of life and it's equally distributed between male and female. They undergo malignant transformation in less than $1 \%$ of cases.(2)

In a study carried out in 2002, it was noted that solitary Enchondromas occurred most commonly in the proximal phalanx of the little finger.(3)

There is paucity of reports on pelvic enchondroma.

We present a rare case of Enchondroma located in the right superior pubic ramus, extending inwards into the pelvic cavity and compressing on the vagina and the rectum, also contributing to infertility.

\section{Case Presentation}

The case is that of a 32 year old nulliparous, who had been married for 4 years and was first seen at the gynaecology clinic of our hospital where she complained of difficulty with conception and deep dyspareunia. On further evaluation she was also found to have a painless pelvic swelling which she noticed while douching. Due to severe excruciating pain during sexual intercourse, she has been avoiding her husband for nearly 6 months. No associated bleeding, vaginal discharge or weight loss. There was associated constipation.

Her general physical examination was essentially normal. She has a normal vulva and vagina, a firm mass was palpated about $6 \mathrm{~cm}$ from the introitus, irregular, lobulated and rough but not tender and continuous with the pubic bone. The mass was about $8 \mathrm{~cm}$ by $6 \mathrm{~cm}$ in its dimension and surrounding tissue appeared healthy. The overlying mucosa was freely mobile. There was no contact bleeding. Digital rectal examination corroborated the vaginal findings.

Her haemograms and erythrocyte sedimentation rate were essentially normal. The patient could not afford Magnetic resonance imaging however pelvic x-rays done, revealed a heterogeneous, hazy opacity of the right superior pubic ramus about $2 \mathrm{~cm}$ from the pubic symphysis, with some calcifications seen within it extending into the pelvic cavity. 
Figure 1 below shows the pelvic x-ray of the patient.

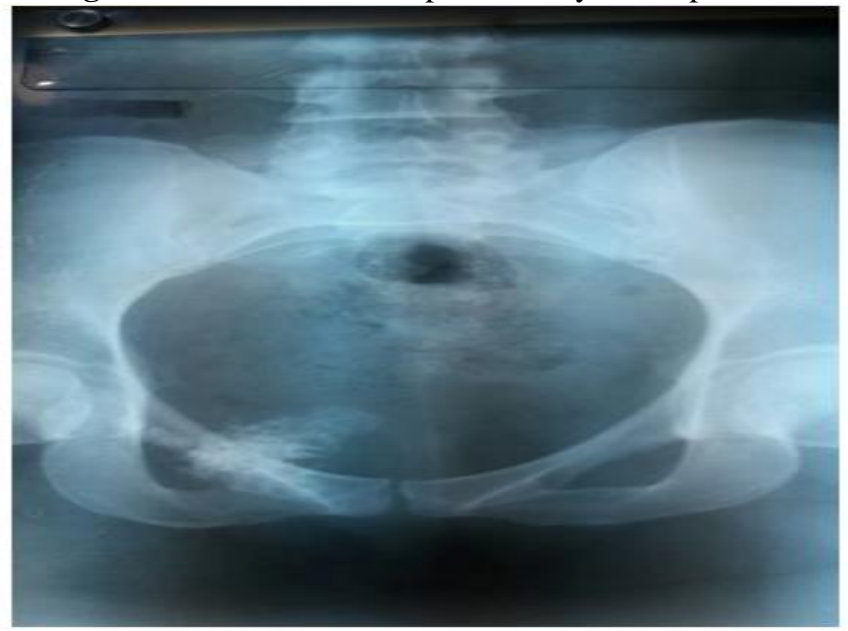

She had excision biopsy done through the abdominal route using Maylard incision. The tumour was approached through extra-peritoneal access taking care not to traumatise the urinary bladder and the bladder neck. The mass appeared cartilaginous in morphology, measuring about four times the size previously determined clinically and radiologically. The mass was seen attached to the right superior pubic ramus and extending medially to the pubic tubercle, laterally extending along the pubic ramus. Inferiorly the mass extended into the pelvic cavity. Piecemeal resection was done and access to the inferior margins of the tumour was gained by osteotomising part of the right superior ramus. Haemostasis was secured with bone wax and fixation done with a reconstruction plate and screws. Histology revealed an Enchondroma with no feature of malignancy. She is currently being followed up in the orthopaedic and gynaecology outpatient departments.

Figures 2 shows the clinical photograph of the tumour after resection.

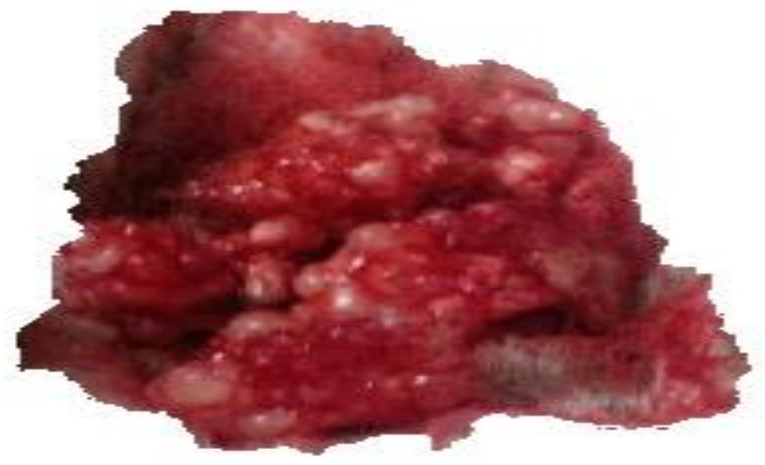

Figure 3 shows the postoperative x-ray

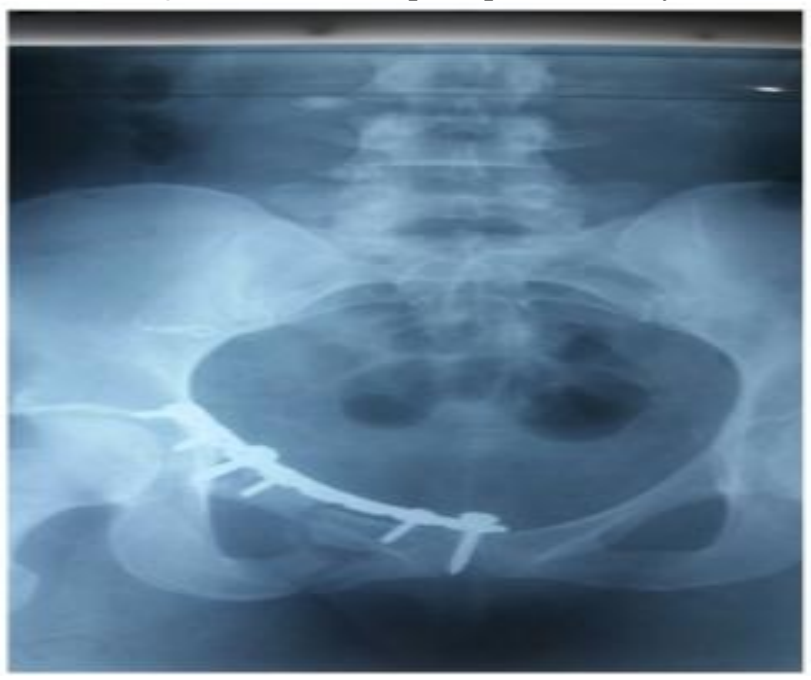




\section{Discussion}

This case report became important due to rare occurrence of this tumour in the pelvis. It is also an uncommon cause of dyspareunia and infertility making it important for discourse. Enchondromas are common in the second to fourth decade of life; the index case therefore falls within this range. The location in the index case has not been reported in literature available within the authors' reach. It is also important to note that during evaluation for dyspareunia, in addition to other modalities of investigation, plain pelvic X-ray may be rewarding.

Furthermore, encroachment of the mass into the pelvic cavity towards the vagina will expectedly cause difficulty in penetration during sexual intercourse this may contribute to the infertility this patient presented with. Deep dyspareunia and subsequent apareunia have been documented in literature as risk factors for infertility. The co-management of the gynaecologists and orthopaedic surgeons was useful in addressing her symptom surgically. The abdominal route was preferred due to proximity of the superior pubic ramus to the abdomen than the perineal approach. Extra-peritoneal approach was considered in order to avoid intra-peritoneal seedling with tumour cell hence the use of Maylard incision. The standard of care for chondromas include curettage alone (4) and or combination of curettage and internal fixation if the bone continuity is breached.(5) In a 20 year follow up study by $\mathrm{Al} \mathrm{JBe}$, only one out of 21 patients developed recurrence.(6) This influenced the mode of management offered this woman.Maintaining pelvic stability via the use of reconstruction plate allows early weight bearing and avoids attendant complications of prolonged immobilization. Also the internal fixation helps maintain the preoperative dimension of the pelvic inlet and outlet preventing pelvic contraction and the sequele.

\section{Conclusion And Recommendation}

The causes of dyspareunia may not be only soft tissue disorders; therefore plain X-Ray may be considered early in screening for possible bony aetiology. A high index of suspicion and plain X-ray or imaging modalities will allow early detection of causative or contributory bony lesions.

Pelvic Enchondroma is a rare entity that may cause infertility in females. In the event of a similar case, our mode of management and line of thought may be beneficial and afford good outcome.

\section{References}

[1]. Flemming DJ, Murphey MD. Enchondroma and Chondrosarcoma. Seminars in Musculoskeletal Radiology. 2000;4(1):0059-72.

[2]. Milgram, .W J. The origins of Osteochondromas and Enchondromas. Clinical Orthopaedics and Related research. 1983.

[3]. Gaulke R. The distribution of solitary enchondromata of the hand. Journal of hand surgery. 2002;27(5):444-5.

[4]. Wulle C. Treatment of endochondromas of the hand. Journal of hand surgery. 1990;15:320-30.

[5]. al JBe. Enchondromas of the hand: treatment with curretage and cemented internal fixation. Journal of hand surgery. 2002;27(5):870-5.

[6]. al LMMe. Enchondroma in the hand- retrospective study- recurrence cases. Journal of hand surgery. 2002;7(1):7-10.A 